time periods at the calendar cut point, the 9992 job/time periods were assigned their relevant expert/group/time period estimate. Classification and regression tree (CART) models were developed to predict each expert's expected assignment, based on previous decisions, to assign estimates for jobs in groups that expert had not assessed and for jobs requiring further review.

Results In preliminary analyses, CART models predicted 91$96 \%$ of the experts' pre-1995 estimates and $77-96 \%$ of $\geq 1995$ estimates. CART estimates were assigned to $3-48 \%$ of the job/ time periods, varying by expert. Overall, $92 \%$ of the job/time periods were assigned the same estimate by at least two experts. Conclusions Our framework reduced the number of exposure decisions needed from each expert compared to job-by-job assessment. Future work will use CART models to identify differences between experts to be resolved and incorporate frequency and intensity of lead exposure estimates.

\section{NIGHT SHIFTWORK AND BREAST CANCER SURVIVAL IN DANISH WOMEN}

Johnni Hansen. Danish Cancer Society Research Center, Copenhagen, Denmark

10.1136/oemed-2014-102362.80

Objectives There is mounting evidence that night shiftwork may increase the incidence of female breast cancer. The influence of night shift work on survival of breast cancer has, however, not been reported. The aim of the present study is to elucidate breast cancer survival in different types of former non-day shiftworkers compared to day-workers.

Method In total 1157 women (23\% nurses), aged less than 75 years, diagnosed with breast cancer (2000-2004) participated in two independent nationwide case-control studies on night shiftwork. Information on the entire work life, including night shiftwork and potential risk factors for breast cancer (e.g. reproduction, BMI, alcohol, HRT, heredity and diurnal preference) was obtained by telephone interviews. All study subjects were followed up for death in the National Cause of Death Register until end of 2011.Cox proportionate hazard models and Kaplan-Meier survival plots were used to perform time-to-event analyses.

Results In total 127 breast cancer cases (11.0\%) had died from this disease at end of follow-up (median follow-up 12.6 years). There was a significant tendency of decreasing survival of breast cancer among both fixed and rotating nightshifts workers compared to daytime shiftworkers and by increasing years of prior non-day time work $(p=0.04)$. Evening workers had about same survival as day workers. The results were only slightly affected by confounders.

Conclusions These data suggest that night shift work prior to breast cancer seems to decrease survival. The association was not strongly modified by lifestyle factors.

\section{THE IMPACT OF RESPIRABLE DUST AND RESPIRABLE QUARTZ ON PULMONARY FUNCTION - RESULTS OF A LONGITUDINAL STUDY}

Matthias Möhner, Norbert Kersten, Johannes Gellissen. Federal Institute for Occupational Safety and Health (BAuA), Berlin, Germany

\subsection{6/oemed-2014-102362.81}

Objectives The present study sought to examine the long-term effects of exposure to respirable dust, in particular of respirable quartz on pulmonary function.

Method The study is based on the Wismut cohort of former uranium miners. Spirometric data, including forced expiratory volume in 1s (FEV1) and forced vital capacity (FVC) were ascertained together with quantitative estimates of cumulative exposure to respirable dust and respirable quartz for each of the 1421 study subjects born between 1954 and 1956. Linear mixed regression models were fitted to identify significant determinants of longitudinal changes in lung function parameters. Point estimators and confidence intervals for the exposure concentration threshold value were fitted by partial likelihood profiles of the corresponding models.

Results Overall, 7122 data records were included in the analysis - on average five spirometries for each miner. The mean annual exposure concentration to respirable quartz was $0.072 \mathrm{mg} / \mathrm{m}^{3}$. It was shown that cumulative exposure to $1 \mathrm{mg} / \mathrm{m}^{3}$-year respirable quartz leads, on average, to a relative reduction in FEV1 of $2.07 \%$ and in the quotient of FEV1/FVC of $2.75 \%(\mathrm{p}<0.001)$. The analysis of the whole respirable dust shows, that the fraction of quartz in the dust is the decisive determinant for the impact of dust. A significant improvement of model fit by applying threshold models could not be observed.

Conclusions This study adds further evidence on the long-term effects of exposure to respirable quartz. Current exposure limits for respirable quartz require a critical review.

\section{THE LUNG BURDEN OF ASBESTOS FIBRES (AF) AND ASBESTOS BODIES (AB) AND THE RISK OF MESOTHELIOMA (MM) FOR EXPOSURES CEASED 30 YEARS AGO}

${ }^{1}$ Enzo Merler, ${ }^{1}$ Paolo Girardi, ${ }^{2}$ Pietro Luigi Barbieri, ${ }^{1}$ Vittoria Bressan, ${ }^{3}$ Anna Benedetta Somigliana. 'Mesothelioma Registry of Veneto Region, Occupational Health Unit, Local Health Authority, Padua, Italy; ${ }^{2}$ Mesothelioma Registry, Occupational Health Unit, Local Health Authority, Brescia, Italy; ${ }^{3}$ Centre of Electronic Microscopy, Lombardy Environmental Protection Agency, Milan, Italy

\subsection{6/oemed-2014-102362.82}

Objectives To estimate the risk of $\mathrm{MM}$ according to $\mathrm{AF}$ and $\mathrm{AB}$ in the lungs.

Method Freeze dried lung samples from 309 MM and 41 controls have been analysed for AF (Scanning Electronic Microscopy) and AB (Optical Microscopy) from subjects investigated and classified for probability and circumstances of asbestos exposure. Odds Ratios (OR) were obtained using logistic regression.

Results $254(82 \%)$ MMs have been classified as occupationally and 25 (8\%) as non-occupationally exposed: Geometric Mean (GM) for AF burden was 1950000 and $608000 \mathrm{ff} / \mathrm{g}$ dlt, respectively; and 39300 and 3300 for $\mathrm{AB} .75 \%$ and $58 \%$ of the AF respectively were amphibole.

Controls reported a GM of $269000 \mathrm{AF}$ and 28 of AB g/dlt.

For any increase of $100.000 \mathrm{ff} / \mathrm{g} \mathrm{dlt}$, we computed an OR of 1.7 (1.3-2.3) for amphibole, 1.1 (1.0-1.3) for chrysotile, among occupational MMs; an OR of $1.3(1.0-1.7)$ and $1.1(1.0-1.1)$ among non-occupational MMs.

The 1997 Helsinki criteria for attribution to occupational exposure would have excluded more than $30 \%$ of MMs under study: here occupational exposures ceased on average 26 years before the disease, and therefore clearance and time since last exposure must be taken into account because are relevant determinants of the retained amount of fibres.

Conclusions The risk of $\mathrm{MM}$ increases with the amount of retained amphibole, and to a lesser extent, of chrysotile fibres. Because occupational and non-occupation asbestos exposures have been to mixture of fibres, the lungs of MM patients are still loaded with amphibole AF. 